\title{
Various manifestations of the outflow connected to the cometary nebula GM 3-12 (RNO 124)^
}

\author{
T. A. Movsessian ${ }^{1}$, T. Yu. Magakian ${ }^{1}$, J. Boulesteix ${ }^{2}$, and P. Amram ${ }^{2}$ \\ 1 Byurakan Astrophysical Observatory, Aragatsotn prov. 378433, Armenia \\ and Isaac Newton Institute of Chile, Armenian Branch \\ e-mail: tigmag@sci.am \\ 2 Observatoire de Marseille, Place Le Verrier 2, 13248 Marseille Cedex 4, France
}

Received 4 February 2003 / Accepted 3 September 2003

\begin{abstract}
We present the results of imaging and scanning Fabry-Pérot interferometry of the cometary nebula GM 3-12 (RNO 124) and Herbig-Haro object HH 376A. The nebula is cone-shaped with the star IRAS 20359+6745 at the apex, straight walls and two probable helical arms. HH 376A is the bow shock structure in the collimated high velocity flow, directed by the axis of the cone nebula, with shock velocity about $70-80 \mathrm{~km} \mathrm{~s}^{-1}$. The spatial and kinematical separation between bow shock and Mach disk in HH 376A was not detected, which makes this object similar to HH 111. The H $\alpha$ profiles in the vicinity of the source are double peaked. The high velocity component can be attributed to the collimated flow.
\end{abstract}

Key words. ISM: individual objects: RNO 124 - ISM: Herbig-Haro objects - ISM: kinematics and dynamics

\section{Introduction}

It is now well known that young stellar objects of different masses and luminosities can act as sources of HerbigHaro (HH) objects, jets or molecular outflows (Eislöffel 1996; Padman et al. 1997; Reipurth \& Bally 2001). A number of these objects are connected to reflection nebulae of different shapes: fan-like, bipolar or comet-like (Mundt et al. 1987). The existence of these so-called cometary nebulae $(\mathrm{CN})$ can be considered as an indicator of youth stars.

The shapes of these nebulae result from the reflection of light of their sources, which is directed by the circumstellar disks. When jets or $\mathrm{HH}$ objects are found in their vicinities, they are always located on the axes of $\mathrm{CN}$, and this is another argument for the connection between the disks and $\mathrm{CN}$. Low velocity weakly collimated molecular outflows, which are also observed near young stellar objects, can affect the shapes of $\mathrm{CN}$. In certain cases, such outflows could blow out the ambient material and produce a cavity along the axis of the nebula (Reipurth et al. 2000).

During an ongoing imaging survey of the nebulous objects in dark clouds, conducted at the Byurakan Observatory and aiming to search for new HH outflows and the analysis of

Send offprint requests to: T. A. Movsessian, e-mail: tigmov@bao.sci.am

* Based on the observations collected with the $2.6 \mathrm{~m}$ telescope of the Byurakan Astrophysical Observatory (BAO) of the National Academy of Sciences of Armenia. nebular morphology, our attention was attracted by GM 3-12 (Gyulbudaghian \& Magakian 1977), also called RNO 124 (Cohen 1980). This small cometary reflection nebula, located in dark cloud L1152, is associated with the star identified as the class I source IRAS $20359+6745$. On the axis of the nebula, 2.6' from the source, a rather bright Herbig-Haro object HH 376A is located, found by Reipurth et al. (1997). We selected this system for a more detailed morphological and kinematical study because its unusual appearance on our images suggested the presence of helical arms.

MHD models of the outflows from YSOs (Shibata \& Uchida 1990; Ouyed et al. 2002) predict formation of several modes of wave structures inside the outflows. These structures look like helices with various opening angles and periods. Small period helices are formed near the axis of the flow and large period ones ar formed in the outer cone.

The first observational evidence of such forms was the finding of a spiral structure in the jet from the L1551 IRS5 source (Itoh et al. 2000), revealed by high spatial resolution observations with the SUBARU telescope. The morphology of several reflection $\mathrm{CN}$ also have helical structures as in the case of prototypic CN, NGC 2261 (Lightfoot 1989; Close et al. 1997; Movsessian \& Magakian 1999). Sometimes such helical structures are of a composite emission-reflection nature, as in the case of RNO 15 (Movsessian \& Magakian 1999), probably excited by oblique shocks on the dense structures. Thus, RNO 124 also could belong to this rare subclass of $\mathrm{CN}$. 


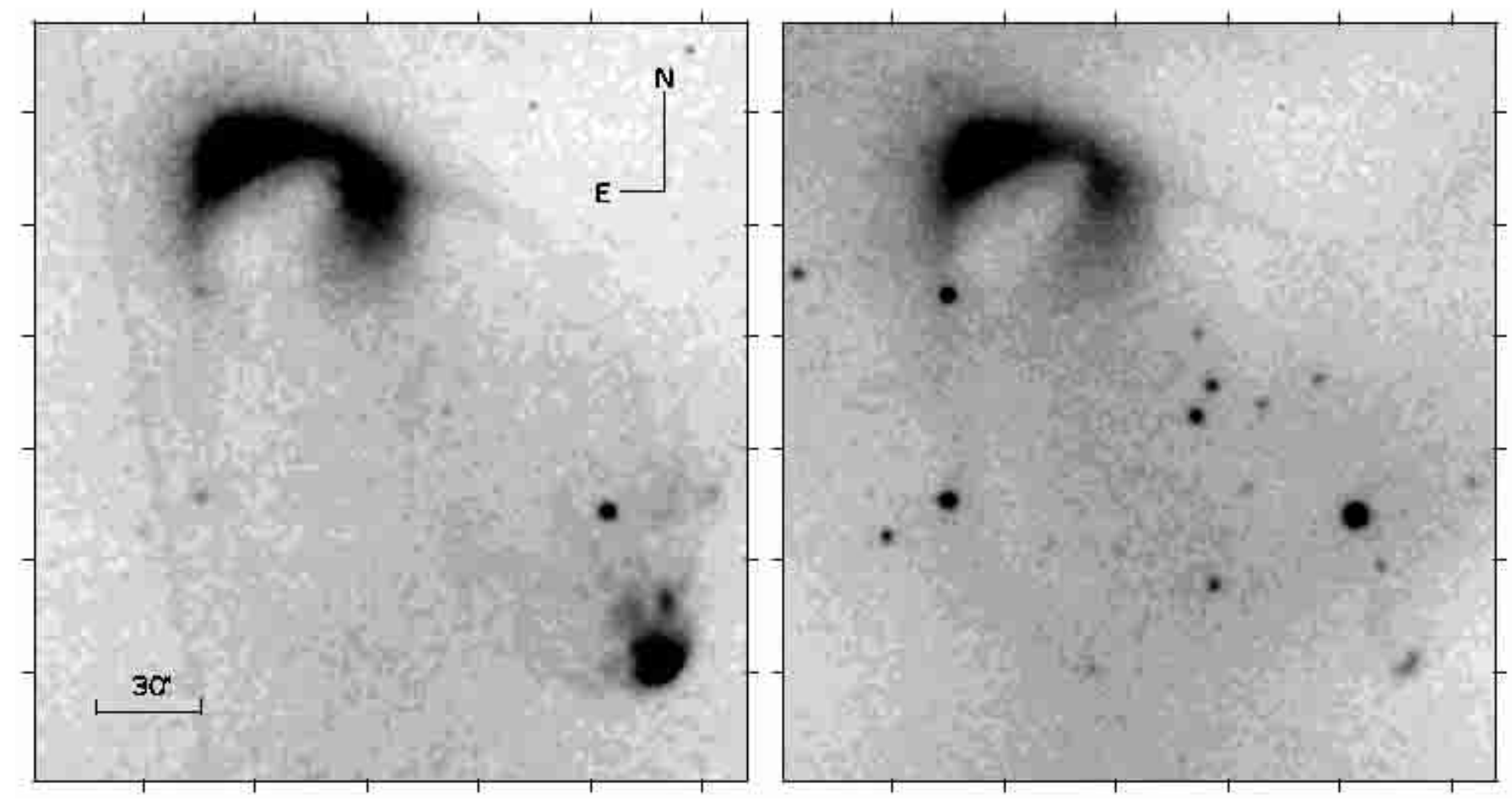

Fig. 1. Deep images of RNO 124 in H $\alpha$ (left) and [S II] (right) lines. The straight walls of the reflection cone and two arm-like structures crossing the cone are clearly seen. HH 376A is the patch bright in $\mathrm{H} \alpha$ in the right lower corner.

\section{Observations and data reduction}

Imaging and Fabry-Pérot (FP) interferometry observations were carried out with the $2.6 \mathrm{~m}$ telescope of the Byurakan Observatory on June 21, 1998, with the ByuFOSC2 (Byurakan Faint Object Spectral Camera, Movsessian et al. 2000b) attached at the prime focus of the telescope. This instrument is a modification of ByuFOSC (Movsessian et al. 2000a).

Narrow band images were obtained with interference filters around [S II] $\left(\lambda_{\mathrm{c}}=6725 \AA, \Delta \lambda=75 \AA\right)$ and $\mathrm{H} \alpha\left(\lambda_{\mathrm{c}}=6565 \AA\right.$, $\Delta \lambda=80 \AA)$. The $I$ filter $\left(\lambda_{\mathrm{c}}=8500 \AA, \Delta \lambda=1200 \AA\right)$ was used for the imaging in continuum. Integration times were $2400 \mathrm{~s}$ in [S II], $1800 \mathrm{~s}$ in $\mathrm{H} \alpha$ and $300 \mathrm{~s}$ in the I-band.

The $\mathrm{H} \alpha$ emission line was scanned with a spectral sampling of $17 \mathrm{~km} \mathrm{~s}^{-1}$ with the scanning Fabry-Pérot interferometer. The exposure time was $420 \mathrm{~s}$ per channel, providing a total exposure time of $2 \mathrm{~h} 48 \mathrm{~m}$ for 24 scanning steps. The quality of seeing was about $2^{\prime \prime}$. The $\mathrm{H} \alpha$ line was isolated with a narrow-band interference filter $\left(\lambda_{c}=6562 \AA, \Delta \lambda=10 \AA\right)$. The Fabry-Pérot interferometer was used in the 787 interference order, which gives $R=18000$ at $\mathrm{H} \alpha$ wavelengths, with a free spectral range of $376 \mathrm{~km} \mathrm{~s}^{-1}$ (or $8.25 \AA$ ) with a sampling of $0.34 \AA$ per channel. The field of view of this system was about $5.5^{\prime} \times 5.5^{\prime}$, which allowed us to study simultaneously both HH 376A and the area near the source star.

The data were reduced in the way described in Movsessian et al. (2000a). Using calibration rings obtained from the $6598.95 \AA$ neon emission line, we established a "phase map", providing the wavelength calibration for each pixel. After the construction of the final wavelength cube the maps of the field at each wavelength as well as in the integral $\mathrm{H} \alpha$ emission were produced.

\section{Results}

\subsection{Direct imaging}

On the red DSS-2 images GM 3-12 has appearance of a star located on the edge of nebular semi-ring. HH 376A object can be barely seen as a small diffuse knot. Our deep images reveal much more detail (Fig. 1). As a whole, this nebula has a conical shape, with a star at its apex. It is possible to separate two distinct reflection structures in the nebula. The former is represented by a regular cone with an opening angle of about $60^{\circ}$ and with straight walls, which are extended up to $2^{\prime}$. This cone sharply stands out from the background and from the scattered emission inside of the nebulous cone. It should be noted that the existence of several background stars near the axis of the cone indicates the presence of a cavity where the matter is blown out by the outflow from the central source as in the case of $\mathrm{LkH} \alpha 234$ (Bertout 1987). The latter structure has the appearance of two spiral arm-like structures crossing each other. The eastern arm is brighter than the western one, which is barely seen on our deep images. This difference could be the result of a greater extinction for the backside of the cone. These features look like two helices wound around the regular concave nebula.

The structure of the nebula is presented in Fig. 2, where we show the set of photometric cuts, perpendicular to the axis of the nebula. Both arms and the cavity walls are marked by dashed lines, which were traced through the local intensity maxima.

Our images also confirm the bow-like shape of HH 376A (Reipurth et al. 1997) and reveal more morphological details. In Fig. 3 a contour plot of $\mathrm{HH} 376 \mathrm{~A}$ in $\mathrm{H} \alpha$ is shown. Several 


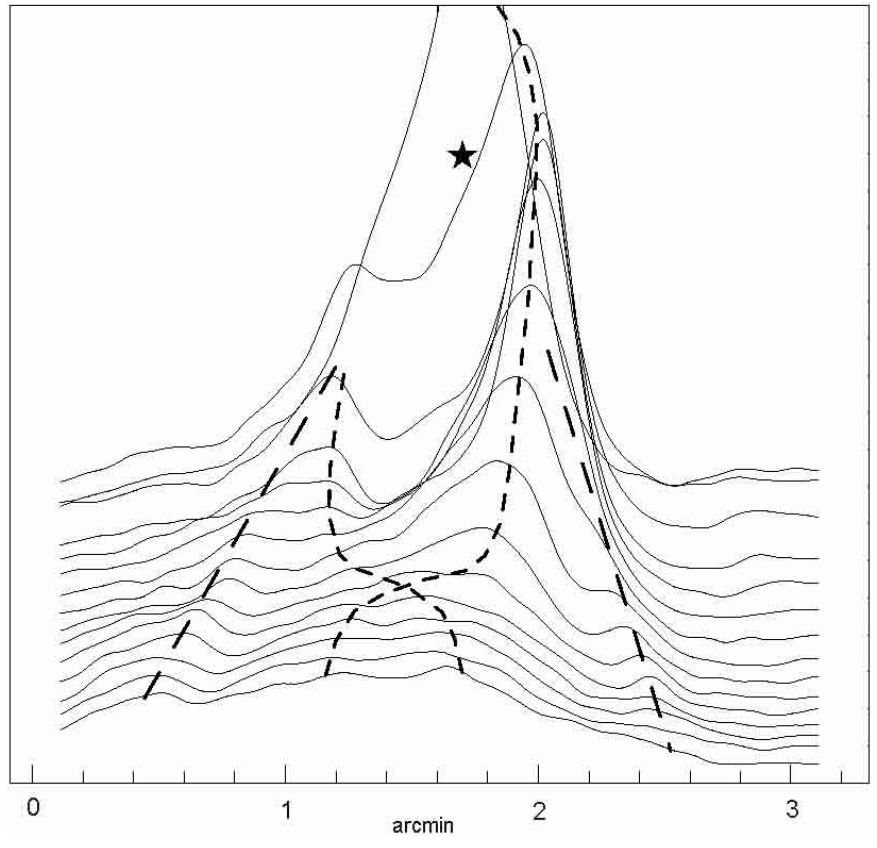

Fig. 2. The schematic presentation of the RNO 124 morphology, overlayed on the set of photometric cuts over its image in the $\mathrm{H} \alpha$ line. Long-dashed lines show the position of the cone walls, and shortdashed ones trace the structures inside the nebula. For convenience the image is rotated to bring the symmetry axis of the nebula into the vertical position. Cuts were taken with 6.3" spacing and averaging through the smoothed and star-subtracted image. The approximate position of the source is marked by an asterisk.

well-defined knots, embedded in the faint amorphous structure, can be seen; they are labelled "a-d". The object has a sharp edge on the side opposite to the source and a more extended internal structure behind it. It is natural to assume that the object moves in the south-western direction and the sharp edge is its leading side. In [S II] HH 376A is much fainter, only the bright head of the bow-shaped structure is seen. There is no evidence of perceptible displacement, i.e. exceeding 1.5-2" between [S II] and $\mathrm{H} \alpha$ images. The estimated ratio of $\mathrm{H} \alpha /[\mathrm{S} \mathrm{II}] 6716+6731 \AA$ for knot "a" is about 3.2. As other knots are not visible in [S II], we can assume that this value is the lower limit for the $\mathrm{H} \alpha /[\mathrm{S}$ II $] 6716+6731 \AA$ ratio for $\mathrm{HH} 376 \mathrm{~A}$ as whole.

\subsection{Fabry-Pérot interferometry}

Interferometric observations enable us to study the velocity field and profile variations of the $\mathrm{H} \alpha$ line across the object. In Fig. 4 the profiles of the $\mathrm{H} \alpha$ line are superposed on the monochromatic image of HH 376A. These profiles are symmetric and no strong variations across HH 376A can be detected. The only significant change of width is seen near the leading edge of HH 376A (Fig. 5), located on its south-western side, as is described in the previous section. In this area the maximal values of FWHM $(2.8 \AA)$ are detected, while in the rest of the object, the FWHM is about $1.6 \AA$. The mean heliocentric velocity of the object is $-105 \mathrm{~km} \mathrm{~s}^{-1}$. There are no prominent variations of radial velocity across HH 376A,

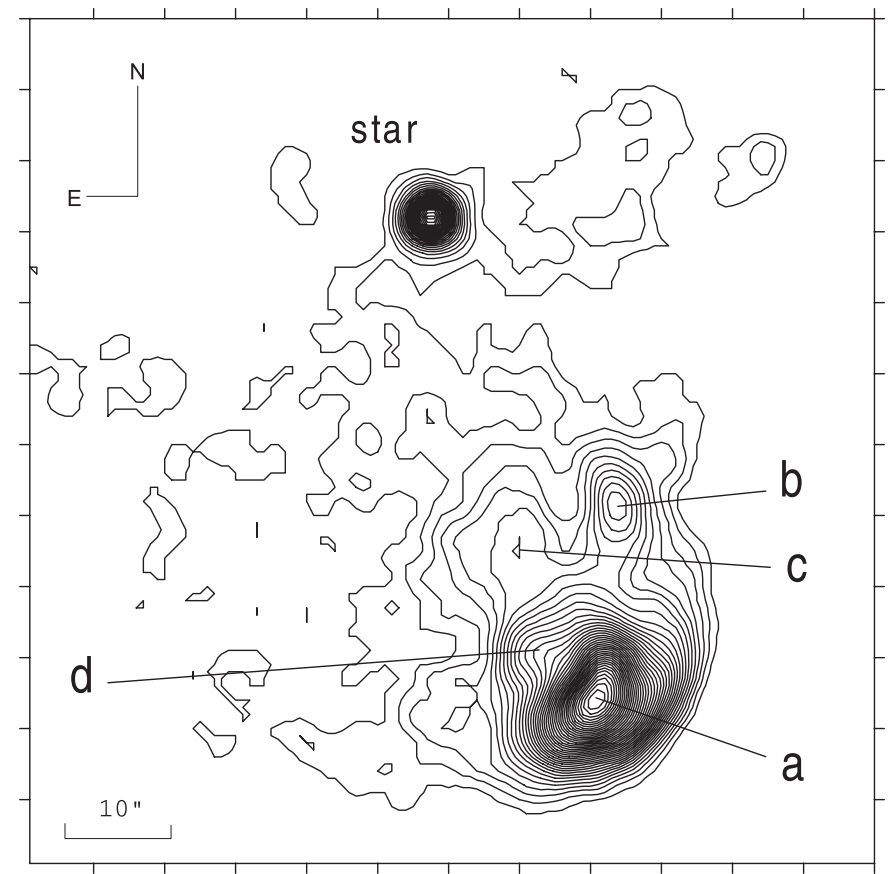

Fig. 3. The contour map of HH 376A in $\mathrm{H} \alpha$ line. Several well-defined knots are indicated.

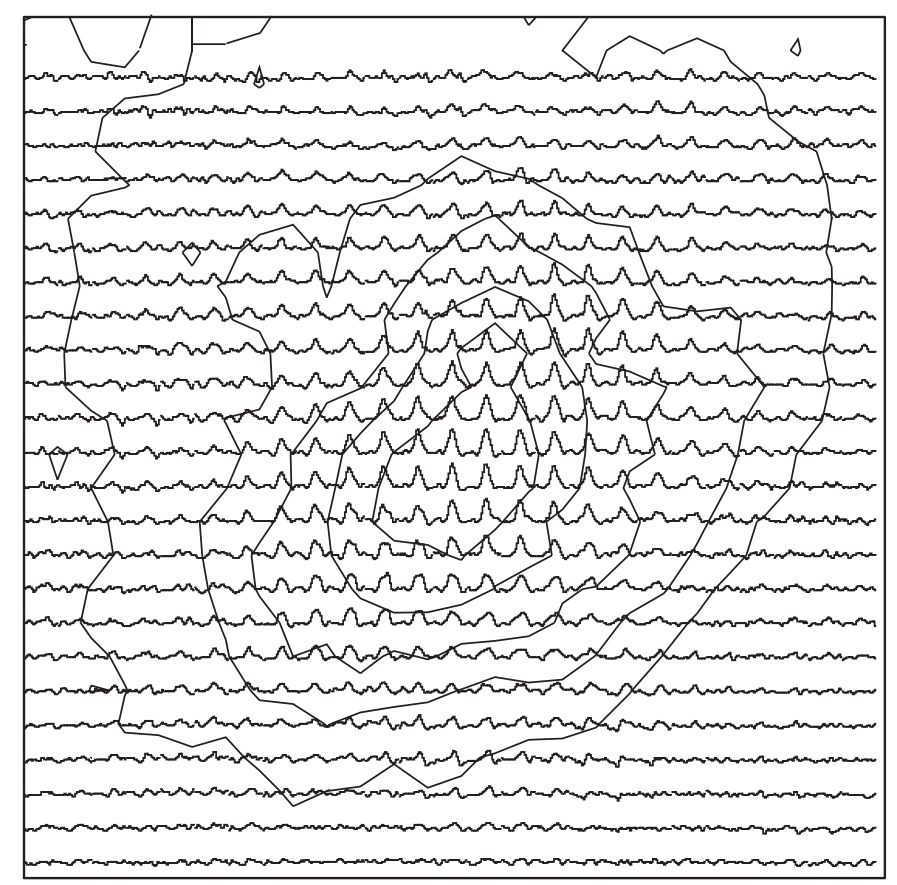

Fig. 4. Spatial grid of $\mathrm{H} \alpha$ profiles superposed on the monochromatic image of HH 376A. Each cell is equivalent to $0.63^{\prime \prime} \times 0.63^{\prime \prime}$ on the sky; thus, the image covers $15.75^{\prime \prime} \times 15.75^{\prime \prime}$. For each profile, the wavelength increases from the left to the right, and covers the range from -310 to $+106 \mathrm{~km} \mathrm{~s}^{-1}$.

except at its leading edge, where the velocity drops to $-72 \mathrm{~km} \mathrm{~s}^{-1}$ (Fig. 6).

One can divide HH 376A into the main body, which does not show the strong variations of FWHM and heliocentric velocity, and to the frontal or leading edge, approaching which 
FWHM $(\mathrm{km} / \mathrm{s})$

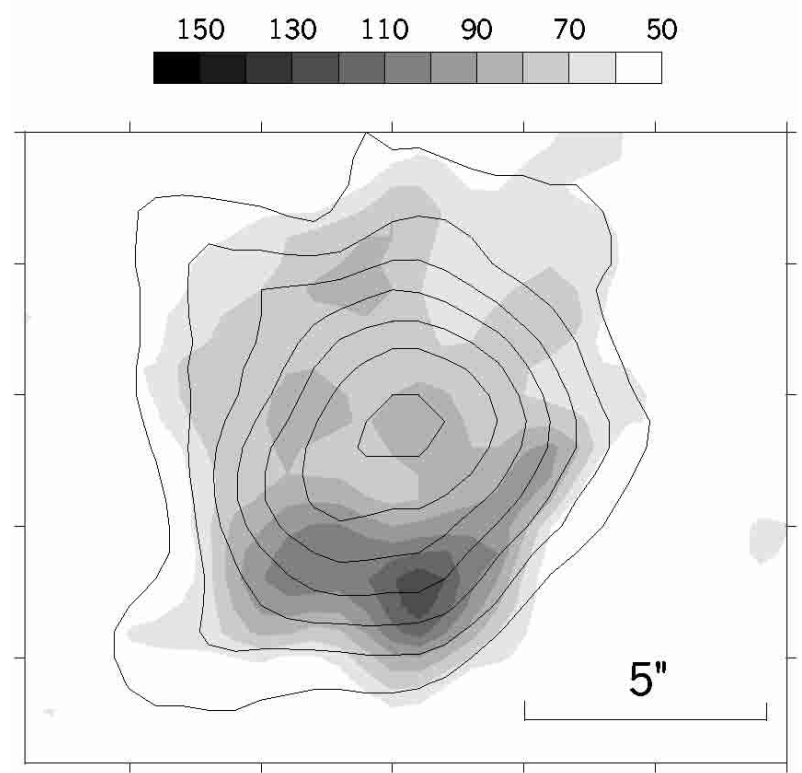

Fig. 5. Map of FWHM of $\mathrm{H} \alpha$ emission with superposed monochromatic image.

we see an increase of FWHM from the center and a sharp drop in radial velocity.

$\mathrm{H} \alpha$ profiles around the source star are presented in Fig. 7. The profiles are double-peaked everywhere, but the low velocity component is stronger in the star itself (ratio $I_{-115} / I_{-25}=0.3$ for the components with the velocities -115 and $-25 \mathrm{~km} \mathrm{~s}^{-1}$ ). Meanwhile the intensities of components in the spectrum of nebula (i.e. in the scattered light) are roughly equal (intensities ratio is $I_{-120} / I_{-15}=0.8$ ). However, our estimates show that the equivalent width of the low velocity component remains the same everywhere. Thus, one can conclude that the intensity of the high velocity component in the scattered light increases.

\section{Discussion}

In Fig. 8 we show the schematic presentation of the RNO 124 system. The helical arms on the walls of the cone and two components of the outflow are shown. One can consider GM 3-12 as a classical cone nebula connected to a PMS star and associated with $\mathrm{HH}$ outflow, as a whole similar to the prototype object, NGC 2261. A large HH object, located on the axis of a $\mathrm{CN}$, represents the region where a high velocity collimated flow (or episodic outburst) collides with a cold dense shell and produces a bow-shaped excitation structure. The above mentioned drop of the extinction inside the nebular cone with well defined walls emphasizes the concave morphology of the object, which is an indicator of the material blowing out along the axis of the object. The instabilities, formed on the walls of this cavity, could produce the observed helical structures. The velocity of the outflow is probably not enough to form the shocked excitation regions; thus these features are observed as reflection structures, similar to those in NGC 2261 (Close et al. 1997).

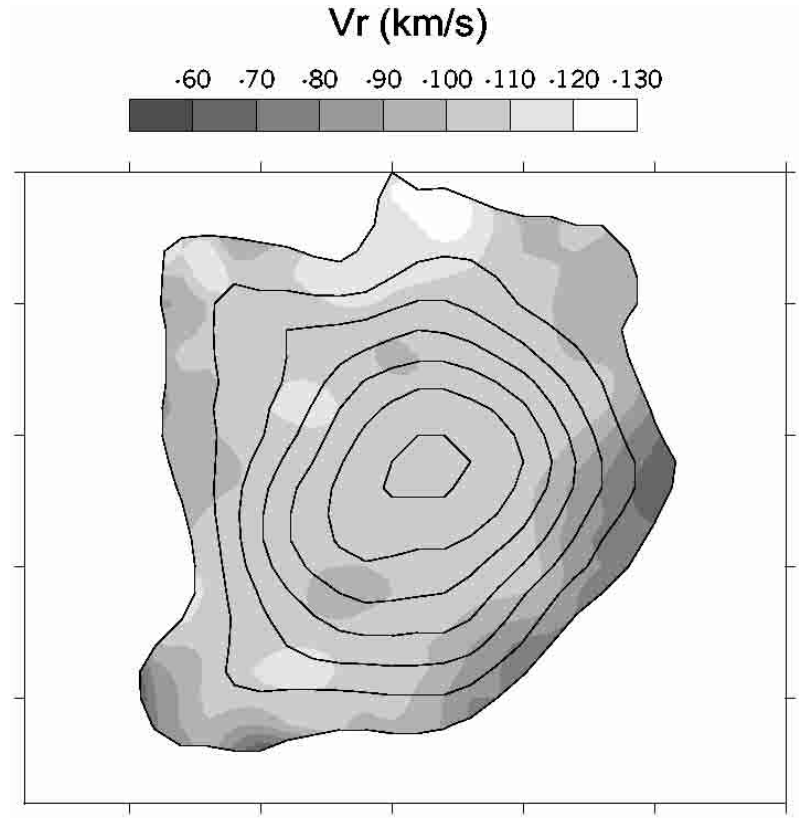

Fig. 6. Velocity field in HH 376A (gray scale) with superposed monochromatic image (isolines). The large velocity gradient at the edge of the bow-shock is prominent.

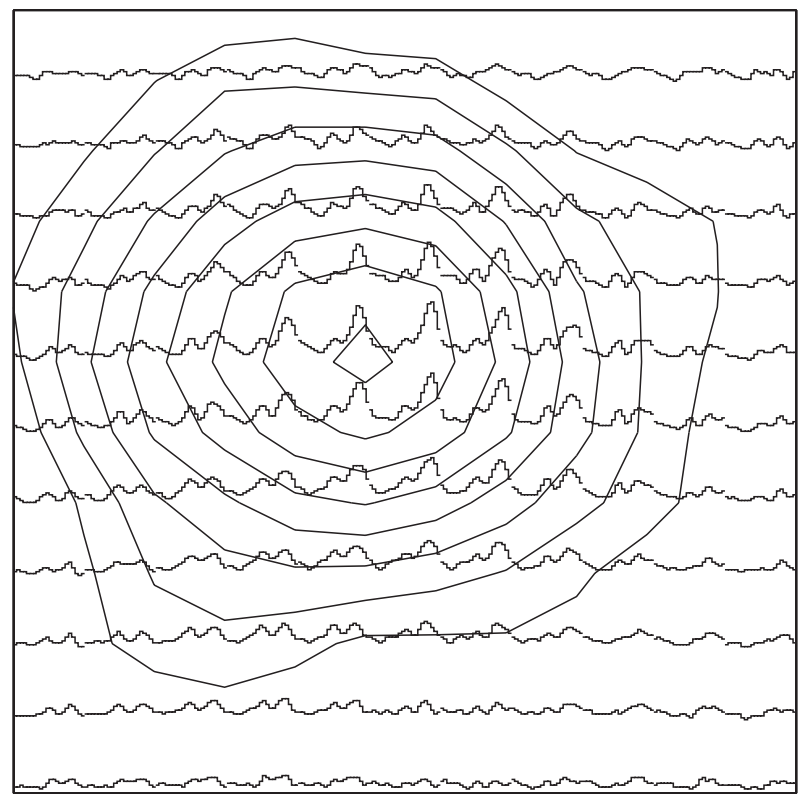

Fig. 7. Spatial grid of $\mathrm{H} \alpha$ profiles superposed on the monochromatic image of the source star of RNO 124. Continuum is subtracted. Each cell is equivalent to $0.63^{\prime \prime} \times 0.63^{\prime \prime}$ on the sky; the covered area is $6.9^{\prime \prime} \times$ $6.9^{\prime \prime}$. Velocity range is same as in Fig. 4.

We do not know yet the full length of the IRAS 20359+6745 flow, because the small group HH 376B, located much further away (10.5' from the source) also could be part of it (Reipurth et al. 1997). HH 376A can be considered as a typical bow-shock of the outflow from IRAS 20359+6745. Taking into account the very slight positional shift between $\mathrm{H} \alpha$ and [S II] images, one can conclude that the two shocked excitation regions (bow shock and Mach disk) here are not spatially separated as clearly as e.g. in the case of $\mathrm{HH} 34$ 


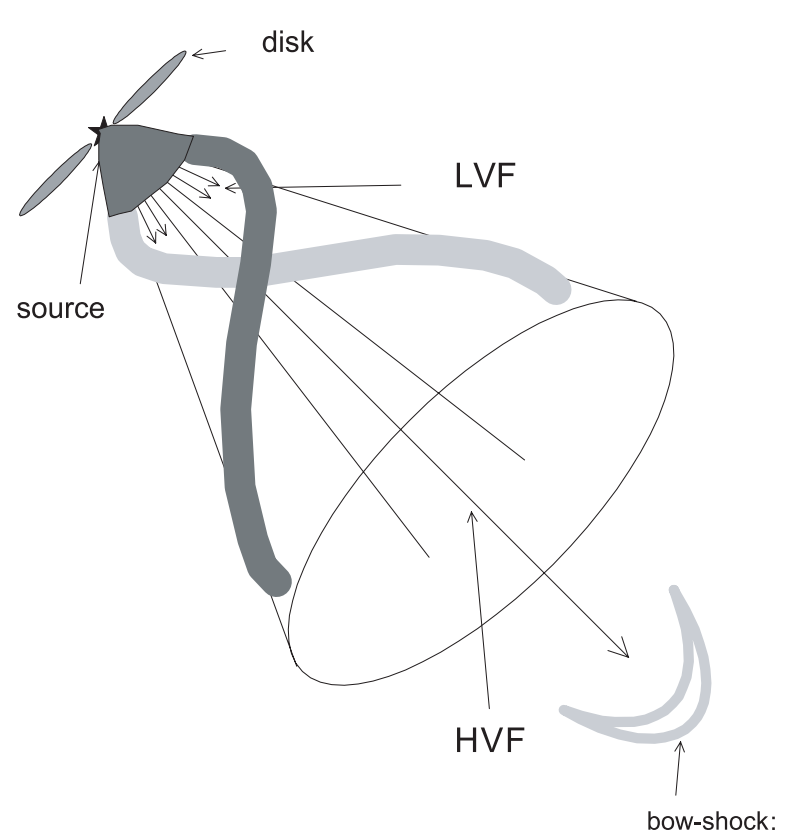

Fig. 8. Schematic presentation of the structure of the RNO 124 system.

(Morse et al. 1992). Thus, this object is more similar to the case of HH 111 (Morse et al. 1993).

This separation (i.e. the cooling distance) is small in the cases where the shock speed is low (see Smith 2003). Projection effects also can lower the visible separation between bow-shock and Mach disk.

The shock velocity on the working surface can be estimated from the FWZI of the emission lines (Hartigan et al. 1987), which is about $150 \mathrm{~km} \mathrm{~s}^{-1}$ for HH 376A. This value is near to the observed radial velocities in $\mathrm{HH}$ 376A (see Fig. 6), taking into account also the inclination of the outflow. But the shock velocity can be lower than the FWZI, because line widths can increase due to several factors, such as shock geometry, thermal broadening and turbulent motion (see Morse et al. 1993, Sect. 4.1). Considering the $\mathrm{H} \alpha /[\mathrm{S}$ II $]$ line ratio, we estimate the shock velocity to be near $70-80 \mathrm{~km} \mathrm{~s}^{-1}$ from planar shock models, described in the works by Hartigan et al. (1987) and Morse et al. (1993). This is consistent with the presumably short cooling distance.

Concerning the variations of the profiles of $\mathrm{H} \alpha$ line in the environment of the source star, one can suggest that the low velocity component represents the emission in the circumstellar envelope of the PMS star. The velocity of the more blueshifted component is close to the velocity of $\mathrm{HH} 376 \mathrm{~A}$, i.e. the outflow velocity; thus this component can be associated with the collimated outflow. Thus, the change of the ratio of the $\mathrm{H} \alpha$ line components in the scattered light, where the high velocity component becames stronger, can be explained by the more significant contribution to the reflected light from the emission of the high velocity moving gas inside the cavity.

\section{Conclusion}

Our observations confirm that RNO 124 is a system with directed outflow, which produces $\mathrm{HH} 376 \mathrm{~A}$ as well as forms the cone reflection nebula with traces of helical arms. The $\mathrm{H} \alpha$ line in the spectrum of the source star shows the presence of a high velocity component, associated with the collimated flow.

Our analysis of the deep images of $\mathrm{CN}$ and of their appearance on the DDS-2 charts leads to the conclusion that the morphological classification of many $\mathrm{CN}$ needs reconsideration, and the existence of probable spiral and helical structures should be taken into account. As was mentioned in Sect. 1, helical structures can be found also in the emission jets. However, to definitely identify these structures as the wave patterns predicted by the theoretical models, further observations are necessary. It seems that 3D spectral methods, such as high spatial and spectral resolution Fabry-Perot and integral-field spectroscopy, will be most efficient for this task.

Acknowledgements. We thank Dr. M.D. Smith for many helpful comments. Part of this work was performed during the stay of one of the authors (T.A.M.) at the Marseille Observatory, made possible through the "Jumelage" cooperation project. We also thank the referee for many valuable critical comments and suggestions. This work was partially supported by INTAS grant 00-00287 and ANSEF grant No. PS103-01.

\section{References}

Bertout, C. 1987, in Circumstellar matter, Proc. IAU Symp. (Dordrecht: D. Reidel), 122, 23

Close, L. M., Roddier, F., Hora, J. L., et al. 1997, ApJ, 489, 210

Cohen, M. 1980, AJ, 85, 29

Eislöffel, J. 1996, in Jets from Stars and Galactic Nuclei (Proceed. Workshop), ed. W. Kundt, Lect. Notes in Phys., 471, 104

Gyulbudaghian, A. L., \& Magakyan, T. Yu. 1977, Astron. Tsirk., 953, 1

Hartigan, P., Raymond, J., \& Hartmann, L. 1987, ApJ, 316, 323

Itoh, Y., Kaifu, N., Hayashi, M., et al. 2000, PASJ, 52, 81

Lightfoot, J. F. 1989, MNRAS, 239, 665L

Morse, J. A., Hartigan, P., Cecil, G., Raymond, J. C., \& Heathcote, S. 1992, ApJ, 399, 231

Morse, J. A., Heathcote, S., Cecil, G., Hartigan, P., \& Raymond, J. C. 1993, ApJ, 410, 764

Movsessian, T., \& Magakian, T. 1999, in Optical and Infrared Spectroscopy of Circumstellar Matter, ed. E. Guenther, B. Stecklum, \& S. Klose, ASP Conf. Ser., 188, 49

Movsessian, T. A., Magakian, T. Yu., Amram, P., Boulesteix, J., \& Gach, J.-L. 2000, A\&A, 364, 293

Movsessian, T. A., Gach, J.-L., Boulesteix, J., Balayan, S. K., \& Zakarian, M. 2000, Baltic Astron., 9, 652

Mundt, R., Brugel, E. W., \& Bührke, T. 1987, ApJ, 319, 275

Ouyed, R., Clarke, D. A., \& Pudritz, R. E. 2002, ApJ, in press

Padman, R., Bence, S. J., \& Richer, J. S. 1997, in Herbig-Haro Flows and the Birth of Low Mass Stars, ed. B. Reipurth, \& C. Bertout, Proc. IAU Symp. (Kluwer), 182, 123

Reipurth, B., Bally, J., \& Devine, D. 1997, AJ, 114, 2708

Reipurth, B., \& Bally, J. 2001, ARA\&A, 39, 403

Reipurth, B., Bally, J., Graham, J. A., Lane, A. P., \& Zealey, W. J. 1986, A\&A, 164, 51

Reipurth, B., Yu, K. C., Heathcote, S., Bally, J., \& Rodriguez, L. F. 2000, AJ, 120, 1449

Shibata, K., \& Uchida, Y. 1990, PASJ, 42, 39

Smith, M. D. 2003, in Jets 2002: Theory and Observations in YSO's (Proceed. Workshop), ed. A. J. L. Fernandes, et al. (Kluwer), in press 